\title{
PECOMA DE COLO UTERINO: UM RARO RELATO DE CASO
}

\section{PECOMA OF THE CERVIX: A RARE CASE REPORT}

Welington Lombardi ${ }^{1}$; Luciana Borges Lombardi ${ }^{1}$; Marina Abud Ferreira ${ }^{2}$; Flávia Vicentin Silva ${ }^{2}$; Silvia Paino Ruas ${ }^{3}$; Fernando Pereira Vanni ${ }^{1}$; Caroline Barbieri Rodrigues ${ }^{4}$; Luana Albaricci Carreon Fernandes ${ }^{4}$.

\section{RESUMO}

Introdução: Os Pecomas são tumores de células epitelióides, de origem mesenquimal, normalmente benignos e extremamente raros. São encontrados mais frequentemente no retroperitônio, em região abdominopélvica, no trato gastrointestinal e no trato ginecológico, podendo ser encontrada também em tecidos moles e ossos. Recentemente foram publicados aproximadamente 100 casos de Pecoma, e cerca de $30 \%$ desses são de origem uterina, sendo o corpo do útero a região mais acometida. Em sua grande maioria possuem comportamento benigno, sendo raro o acometimento de estruturas ou órgãos vizinhos. Relato do caso: Trata-se de uma paciente de 38 anos, que apresentava sangramento uterino persistente, volume uterino aumentado e anemia associada. O ultrassom transvaginal mostrava a presença de vários nódulos sugestivos de leiomiomas. Após tratamento cirúrgico com histerectomia abdominal total, o anatomopatológico confirmou a presença de miomatose uterina, neoplasia intraepitelial cervical grau III e Pecoma de colo uterino. O exame de imunohistoquimica confirmou o diagnóstico de Pecoma, através da positividade para HMB-45, no achado cirúrgico. Conclusão: Portanto, por haver um número pequeno de relatos e estudos sobre o diagnóstico e tratamento do Pecoma, é importante e relevante estudarmos mais sobre essa afecção para melhorar os niveis de evidências e conhecimento sobre tal doença.

Palavras-chave: Neoplasias do Colo do Útero. Imuno-Histoquímica. Diagnóstico. Neoplasias de Células Epitelioides Perivasculares.

\begin{abstract}
Introduction: Pecomas are epithelioid cell tumors, of mesenchymal origin, usually benign and extremely rare. They are found most often in the retroperitoneum, in the abdominopelvic region, in the gastrointestinal tract and in the gynecological tract, and can also be found in soft tissues and bones. Recently, approximately 100 Pecoma cases have been published, and about 30\% of these are of uterine origin, the body of the uterus being the most affected region. Most of them have benign behavior, being rare the involvement of neighboring structures or organs. Case report: This is a 38-year-old patient who had persistent uterine bleeding, increased uterine volume and associated anemia. Transvaginal ultrasound showed the presence of several nodules suggestive of leiomyomas. After surgical treatment with total abdominal hysterectomy, the anatomopathological examination confirmed the presence of uterine fibroids, grade III cervical intraepithelial neoplasia and cervical Pecoma. The immunohistochemistry test confirmed the diagnosis of Pecoma, through positivity for HMB-45, in the surgical finding. Conclusion: Therefore, as there are a small number of reports and studies on the diagnosis and treatment of Pecoma, it is important and relevant that we study more about this condition to improve the levels of evidence and knowledge about this disease.

Keywords: Uterine Cervical Neoplasms. Diagnosis. Immunohistochemistry. Perivascular Epithelioid Cell Neoplasms.
\end{abstract}

\section{INTRODUÇÃO}

Os Pecomas são tumores de células epitelióides, de origem mesenquimal, normalmente benignos e extremamente raros $^{1}$. Foram descritos inicialmente por Apitz, em 1943, especificamente em angiomiolipoma de rim e, depois, em pacientes com tumores pancreáticos ${ }^{2}$. Em 1992 Bonetti e colaboradores denominaram de Pecoma um tipo celular com aparência epitelióide, citoplasma eosinofilico claro e distribuição perivascular, existentes nos angiomiolipomas renais (AML), tumor de células claras do pulmão (TCCP) e

\footnotetext{
${ }^{1}$ Universidade de Araraquara, Departamento de Ginecologia - Araraquara - SP - Brasil

2Universidade de Araraquara, Programa de Ginecologia e Obstetrícia - Araraquara - SP - Brasil

${ }^{3}$ Anatomia Patológica Dr Paino - Araraquara - SP - Brasil

${ }^{4}$ Universidade de Araraquara, Acadêmica da Faculdade de Medicina - Araraquara - SP - Brasil
} 
linfangioleiomiomatose (LAM). Os AML são os mais comuns, com prevalência de $0,13 \%$ e acontecem mais frequentemente no sexo feminino. A prevalência da LAM é duvidosa, mas acredita-se que seja de 1 a 2 pessoas por milhão de habitantes. A prevalência no sexo feminino é exorbitante, enquanto em mulheres existem mais de 1400 casos diagnosticados em homens são somente $4^{3,4}$.

Somente em 2002, a Organização Mundial da Saúde (OMS) reconheceu essa entidade separadamente atribuindo a ela, uma nova classificação5,6. São encontrados mais frequentemente no retroperitônio, em região abdominopélvica, no trato gastrointestinal e no trato ginecológico, podendo ser encontrada também em tecidos moles e ossos 7,8 . Os sintomas apresentados podem ser assintomáticos ou inespecíficos, tais como náuseas, anorexia e queixas álgicas ${ }^{9,10}$.

Recentemente foram publicados aproximadamente 100 casos de Pecoma, e cerca de $30 \%$ desses são de origem uterina, sendo a região mais acometida o corpo uterino, seguida pelo colo. Os Pecomas uterinos afetam pacientes com idades variáveis de 3 a 97 anos, sendo mais frequente em torno dos 40 anos. Os sintomas mais comuns são episódios de sangramento vaginal, sindrome da compressão extrínseca, dor abdominal e aumento do volume uterino $3,11-13$. No entanto, essas manifestações também são comuns em pacientes com outros tipos de tumores uterinos, acarretando dificuldades para o diagnóstico, tanto ao exame clínico quanto aos exames de imagem, sendo indispensável a realização da biópsia ${ }^{14}$.

Do ponto de vista histológico, os Pecomas apresentam citoplasma claro ou granular e se organizam, na maioria das vezes, de forma perivascular, expressando marcadores miogênicos como a actina de musculo liso (SMA) e malanocíticos (HMB45 e Melan-A). Em sua grande maioria possuem comportamento benigno, apresentando-se como uma massa circunscrita, com constituintes sólidos e císticos, sendo raro o acometimento de estruturas ou órgãos vizinhos ${ }^{7}$. Ainda assim, alguns estudos demonstraram comportamento localmente agressivo e metástases a distância para figado, pulmões, intestino, ossos e linfonodos. Nesses casos, apresentavam características morfológicas semelhantes aos Tumores de Açúcar de Células Claras do Pulmão (CCST), angiomiolipomas (AML) e linfangioleiomiomatoses (LAM). Foi também demonstrada forte associação dos tumores localmente agressivos como Complexo da Esclerose Tuberosa (TSC) ${ }^{15,16}$.

Em casos de malignidade, o paciente deve apresentar pelo menos duas das seguintes características: tamanho do tumor $>5 \mathrm{~cm}$, padrão de crescimento infiltrativo, hipercelularidade, núcleos exuberantes e hipercrômicos, zonas de necrose, alta atividade mitótica $(>1 / 50)$, invasão vascular e mitoses atípicas, sendo que as metástases tardias nos ossos, linfonodos, pulmão, intestinos e figado comprovam a malignidade da neoplasia ${ }^{17,18}$.

Para o diagnóstico diferencial são utilizados alguns marcadores sensiveis como o HMB-45 e o Melan-A, que auxiliam na diferenciação entre Melanomas, Carcinomas de Células Claras, Mioepiteliomas, Paragangliomas, Leiomiossarcomas e Sarcomas estromais endometriais $\quad(\mathrm{ESS})^{7,19,20}$. Devido à quantidade reduzida de relatos sobre essa patologia e a ausência de informações suficientes, ainda não existe um consenso na escolha da melhor conduta terapêutica. A cirurgia, porém, continua sendo o tratamento mais indicado para os casos de tumor primário, metástases ou recidiva local, sendo importante o acompanhamento para avaliação da necessidade de um tratamento clínico adjuvante ${ }^{9,15,17}$. Esse trabalho foi submetido ao Comitê de Ética e Pesquisa da Universidade de Araraquara UNIARA, sob o registro CAAE: 40973520.6.0000.5383.

\section{RELATO DO CASO}

L. S., 38 anos, branca, procurou serviço de referência em ginecologia devido a quadro clínico de sangramento uterino persistente e anemia associada. Negava antecedentes pessoais e familiares relevantes. Ao exame físico, apresentava mucosas hipocoradas $(++/++++)$, hidratadas, pulso e pressão arterial normais. Ao toque vaginal, apresentava colo endurecido, lobulado, indolor, impérvio, com útero doloroso à mobilização, aumentado de volume, compatível com aproximadamente 14 semanas de gestação. Trouxe exame complementar de ultrassonografia transvaginal, que 
mostrava útero em anteversoflexão (AVF), com volume de 236,9 $\mathrm{cm}^{3}$ e presença de miomas intramurais e submucosos, sendo os maiores com volumes de 3,5 e $2,6 \mathrm{~cm}^{3}$, respectivamente (Figura 1). Apresentava, ainda, exame laboratorial que mostrava hemoglobina de $8,6 \mathrm{mg} / \mathrm{dl}$.

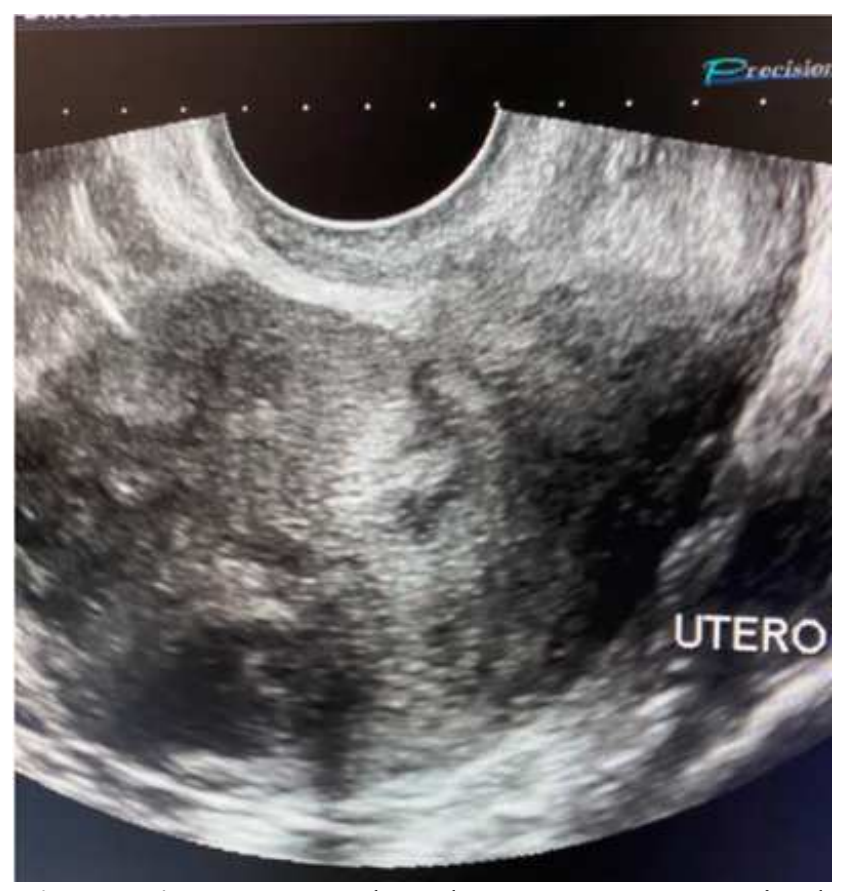

Figura 1. Imagem do Ultrassom transvaginal, mostrando útero aumentado de volume com várias imagens arredondadas e anecóides, sugestivas de miomatose uterina.

Em virtude do quadro clínico apresentado e do exame laboratorial, foi indicado tratamento cirúrgico. A paciente foi, então, submetida à Histerectomia Total Abdominal, a qual ocorreu sem intercorrências.

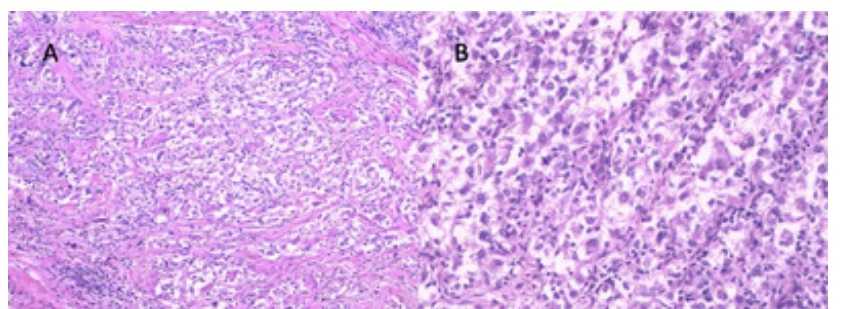

Figura 2. Microscopia mostrando células epitelióides vasculares com citoplasma eosinofílico claro, núcleos e pequenos nucléolos (A. pequeno aumento/B. maior aumento).

O exame anatomopatológico confirmou, além da patologia benigna uterina, com a presença de múltiplos leiomiomas intramurais e submucosos, a presença concomitante de carcinoma in situ de colo uterino e Pecoma de cérvix uterino, localizado na porção muscular lisa do colo, medindo $2,0 \times 1,5 \mathrm{~cm}$, com margens cirúrgicas livres (Figura 2). A imunohistoquímica da lesão do colo uterino revelou positividade para HMB-45, confirmando o diagnóstico de Pecoma (Figura 3).

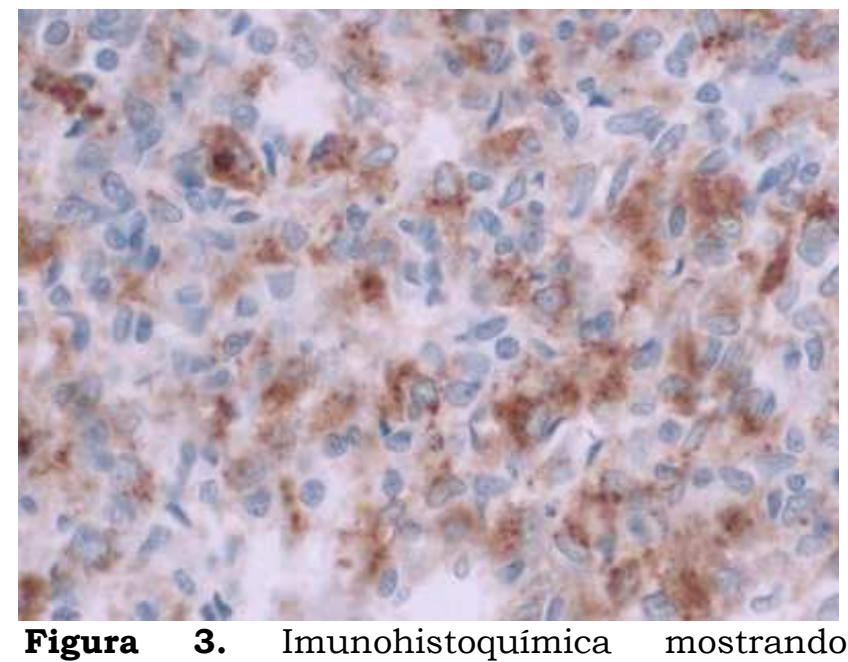
positividade para HMB-45.

\section{DISCUSSÃO}

Os Pecomas uterinos são tumores de células epitelióides raras que apresentam características imuhistoquímicas distintas e que foram categorizados pela primeira vez em 2002, pela OMS5,6. Podem ser classificados em benignos (quando não apresentam características atipicas), potencialmente malignos ou incertos (quando apresentam pleomorfismo nuclear/células gigantes multinucleadas ou possuem tamanho $>5 \mathrm{~cm}$ ), ou malignos (quando apresentam duas ou mais das seguintes características: tamanho $>5 \mathrm{~cm}$, crescimento infiltrativo, alto grau nuclear e de celularidade, mitoses $>1 / 50$ HPFs, necrose e invasão linfovascular) ${ }^{16}$.

A sua origem ainda permanece desconhecida, contudo há estudos que apontam seu surgimento de células-tronco neurais presentes nos revestimentos dos vasos e que poderiam tornar-se capazes de se diferenciar tanto em miócitos quanto em células melanocíticas ${ }^{1-3}$. E a sua relação com possiveis fatores de risco também permanece incerta ${ }^{3}$.

Os Pecomas podem ser confundidos com tumores do músculo liso, já que apresentam morfologia e imunohistoquímica semelhantes. Portanto, é necessário que se realize um diagnóstico 
correto, através de biópsia dirigida e estudo de imunohistoquímica para que a terapia seja realizada de maneira certeira e efetiva ${ }^{6,15,16}$.

Dos casos de Pecoma existentes, cerca de $25 \%$ são de origem ginecológica, a localização mais comum é a uterina, sendo a região mais acometida o corpo uterino, seguida pelo colo. Isso evidencia a raridade do acometimento do cérvix uterino e a importância de se relatar e estudar essa patologia ${ }^{3}$.

No presente estudo a paciente apresentava sangramento uterino anormal, anemia e a ultrassonografia transvaginal mostrava a presença de leiomiomatoses e aumento do volume uterino, levando a um diagnóstico de miomatose uterina, sendo optado por tratamento cirúrgico através da realização de histerectomia abdominal total. Portanto, o diagnóstico de Pecoma de colo foi um achado cirúrgico. $\mathrm{Na}$ maioria das vezes, quando presente, a sintomatologia do Pecoma parece ser inespecífica, como náuseas, anorexia, algias ou, como neste caso, decorrente do local de sua apresentação ${ }^{3}$.

Como o trato ginecológico representa $25 \%$ dos casos de Pecoma e, sendo o corpo uterino o local mais acometido, associado ainda com a presença de múltiplos miomas, seria realmente muito difícil o diagnóstico clínico, já que o mesmo acometia o colo uterino. Em relação a faixa etária, a paciente se enquadra no pico de incidência, encontrado na literatura, para o tumor de células epiteliais perivasculares ${ }^{11,17}$.

No momento do diagnóstico é comum que se confunda o Pecoma com o melanoma convencional devido a expressão de HMB-45 e/ou Melan-A por ambos, bem como características morfológicas semelhantes, como a presença concomitante de células fusiformes e epitelióides, e nucléolo evidente. Com isso, a expressão da proteína $\mathrm{S}-100$ e a não imunorreatividade de actina são importantes para diferenciá-los por ser inerente aos melanomas ${ }^{1,3,12}$.

O tratamento do Pecoma ainda não está bem elucidado, mas em geral, recomenda-se a exérese cirúrgica da lesão, sempre que existir possibilidade de acesso ${ }^{7,9,19}$. Neste caso, optou-se por fazer a histerectomia total abdominal devido a miomatose, sendo o diagnóstico de Pecoma de colo, um achado pós-cirúrgico. Alguns estudos, no entanto, trazem a histerectomia radical com salpingooforectomia bilateral como opção a ser considerada em casos que o Pecoma seja localizado ou disseminado no colo uterino ${ }^{12}$.

\section{CONCLUSÃO}

Pelo fato de haver um número pequeno de relatos, os estudos sobre o diagnóstico e tratamento do Pecoma são escassos e discordantes na literatura. Portanto, seria relevante obter maior número de dados clínicos, moleculares e citogenéticos, bem como o seguimento e controle das pacientes para que assim hajam dados suficientes para recomendações com niveis de evidências confiáveis e unânimes.

\section{REFERÊNCIAS}

1. Montalvão $\mathrm{P}$, Cabrera R, Cruz J, Saraiva J, Santiago N. Pecoma cervical - a propósito de um caso clínico. Port J ORL. 2010;48(4):217-21. doi: $10.34631 /$ sporl.215.

2. Fadare O. Uterine Pecoma: appraisal of a controversial and increasingly reported mesenchymal neoplasm. Int Semin Surg Oncol 2008;5:7. doi: 10.1186/1477-7800-5-7.

3. Rego JL, et al. Pecoma uterino: uma entidade rara. Acta Obstet Ginecol Port. 2014;8(4):385-90.

4. Park J, Seo AN. TFE3-expressing primary perivascular epithelioid cell tumor of the Lymph node mimicking nodal relapse of rectal cancer: A case report. Int $\mathrm{J}$ Surg Case Rep. 2019;59:46-9.

doi: 10.1016/j.ijscr.2019.05.002.

5. Armah HB, Parwani AV. Malignant perivascular epithelioid cell tumor (PEComa) of the uterus with late renal and pulmonary metastases: a case report with review of the literature. Diagn Pathol. 2007;2:45.

doi: 10.1186/1746-1596-2-45.

6. Vang R, Kempson RL. Perivascular epithelioid cell tumor ('PEComa') of the uterus: a subset of HMB-45-positive epithelioid mesenchymal neoplasms with an uncertain relationship to pure smooth muscle tumors. Am J Surg Pathol. 2002;26(1):1-13. 
doi: 10.1097/00000478-20020100000001.

7. Theofanakis C, Thomakos N, Sotiropoulou M, Rodolakis A. Perivascular epithelioid cell tumor of the uterus: Report of two cases and mini-review of the literature. Int $\mathrm{J}$ Surg Case Rep. 2016;28:85-7.

doi: 10.1016/j.ijscr.2016.09.017.

8. Acosta-Materán RV, et al. Perivascular epithelioid cell tumor of the ileum. A case report. Rev Esp Enferm Dig. 2016;108(11):741-2.

doi: 10.17235/reed.2016.3970/2015.

9. Girard FM, Nunes AB, Hauth LA. Malignant subcutaneous PEComa on the cheek. An Bras Dermatol. 2018;93(6):934-5.

doi: 10.1590/abd1806-4841.20187595.

10. Chan YB, Kut E, Koç A. Recurrence of retroperitoneal localized perivascular epithelioid cell tumor two years after initial diagnosis: case report. Sao Paulo Med. J. 2019;137(2):206-8. doi: 10.1590/1516-3180.2017.0120050717.

11. Liu C, Chao W, Lin S, Lau $\mathrm{H}, \mathrm{Wu} \mathrm{H}$, Wang P. Malignant perivascular epithelioid cell tumor in the female genital tract. Medicine (Baltimore). 2019;98(2):e14072. doi: 10.1097/MD.0000000000014072.

12. Kwon BS, Suh DS, Lee NK, Song YJ, Choi KU. Two cases of perivascular diagnostic challenge. Eur J Med Res. 2017;22(1):7. doi: 10.1186/s40001-017-0248-y.

13. Okamoto S, Komura M, Terao Y, Kurisaki-Arakawa A. Respiratory Medicine Case Reports Pneumothorax caused by cystic and nodular lung metastases from a malignant uterine perivascular epithelioid cell tumor (PEComa). Respir Med Case Rep. 2017;22:77-82. doi: 10.1016/j.rmcr.2017.06.011.

14. Fadare O, Parkash V, Yilmaz Y, et al. Perivascular epithelioid cell tumor (PEComa) of the uterine cervix associated with intraabdominal "PEComatosis": aclinicopathological study with comparative genomic hybridization analysis. World J Surg Oncol. 2004:2:35.

doi: 10.1186/1477-7819-2-35.

15. Folpe AL, Mentzel T, Lehr HA, Fisher C, Balzer BL, Weiss SW. Perivascularepithelioid cell neoplasms of soft tis- sue and gynecologic origin: a clinicopathologicstudy of 26 cases and review of the literature. Am J Surg Pathol. 2005;29(12):1558-75. doi: 10.1097/01.pas.0000173232.22117.3 7.

16. Bennett JA, et al. Uterine PEComas: A Morphologic, Immunohistochemical, and Molecular Analysisof 32 Tumors. Am J Surg Pathol. 2018;42(10):137083.

doi: 10.1097/PAS.0000000000001119.

17. Fitzpatrick M, Khalifa M. Perivascular Epithelioid Cell Tumor of the Uterus with Ovarian Involvement: A Case Report and Review of the Literature. Am J Case Rep. 2016;17:309-14. doi: $10.12659 /$ ajcr.896401.

18. Musella A, Felice F, Kyriacou AK, Barletta F, Maria F, Matteo D, et al. Perivascular epithelioid cell neoplasm (PEComa) of the uterus: A systematic review. Int J Surg. 2015;19:1-5. doi: 10.1016/j.ijsu.2015.05.002.

19. Zhao J, et al. Malignant perivascular epithelioid cell tumor of the lung synchronous with a primary adenocarcinoma: one case report and review of the literature. BMC Cancer. 2019;1-5.

20. Papoutsis D, Sahu B, Kelly J, Antonakou A. Perivascular epithelioid cell tumour and mesonephric adenocarcinoma of the uterine cervix: an unknown. 2019;19(1):235. doi: 10.1186/s12885-019-5383-0.

Fonte de financiamento: Não

Conflito de interesses: Não

Data de Submissão: 14 Abril 2021

Decisão final: 11 Agosto 2021

\section{Autor de Correspondência:}

Flávia Vicentin Silva

E-mail: flavia_fvs@hotmail.com 